\title{
Relación entre las normas de género y el consumo de alcohol: una revisión sistemática
}

\section{The Relationship between Gender Norms and Alcohol Consumption: A Systematic Review}

\author{
Rosa María Patró-Hernández*, Yamal Nieto Robles*, Rosa María limiñana-Gras*. \\ * Facultad de Psicología. Universidad de Murcia. España.
}

\section{Resumen}

El consumo de alcohol presenta perfiles diferenciales entre hombres y mujeres, existiendo diferencias igualmente respecto al curso y pronóstico de los problemas derivados del abuso de alcohol. Existe evidencia acerca de estas diferencias en función del sexo, pero la investigación acerca de su relación con diferencias en función de dimensiones asociadas al género es más escasa. Con el objetivo de conocer qué es lo que se ha investigado sobre el tema, se revisa la literatura acerca de la relación entre la conformidad con las normas de género y consumo de alcohol en adultos. Se llevó a cabo una revisión sistemática de la literatura sobre el tema en las bases de datos PubMed, PsycINFO y ScienceDirect. Se incluyeron y analizaron 24 estudios publicados en inglés o español. Los resultados más importantes fueron: 1) la conformidad con normas asociadas al rol tradicional masculino (dominancia, donjuanismo, agresividad, conductas de riesgo) está relacionada, en general, con un mayor consumo de alcohol; 2) la conformidad con normas asociadas al rol tradicional femenino (interés en vida hogareña y cuidado de la familia) se asocia con menor consumo de alcohol. Estos hallazgos proporcionan evidencia acerca de la relación entre dimensiones asociadas al género y el consumo de alcohol. Se considera que las posibilidades de modificación de las creencias y patrones de género vinculados con comportamientos de riesgo es un aspecto a considerar en el ámbito de la prevención, siendo el desarrollo de medidas de género una tarea necesaria para continuar profundizando en el estudio de estas relaciones.

Palabras clave: Normas de género; Masculinidad; Feminidad; Alcohol; Salud.

\begin{abstract}
There are different profiles of alcohol consumption for men and women, and different courses and prognoses associated with problems caused by alcohol abuse. There is evidence of these differences by sex, but research on their links to differences associated with gender dimensions is scarcer. In order to know what has been researched on the subject, this article reviews the literature regarding the relationship between conformity with gender norms and alcohol use and/or abuse in adults. A systematic review was conducted using the electronic databases of PubMed, PsycINFO and ScienceDirect. Twenty-four articles published in English or Spanish were included and analysed. The main findings were: 1) conformity to norms associated with traditional masculine role (dominance, womanising, aggressiveness, risk behaviours) is related to greater alcohol use; 2) conformity to norms associated with traditional feminine role (interest in home life and family care) is related with lower alcohol use. These findings provide evidence of the relationship between dimensions associated with gender and drinking. It is considered that the possibilities of modifying beliefs and gender patterns linked to risk behaviours is an aspect to be taken into account in the field of prevention, with the development of gender measures a necessary task to further deepen the study of these relationships.

Key Words: Gender norms; Masculinity; Femininity; Alcohol; Health.
\end{abstract}

Recibido: Mayo 2018; Aceptado: Noviembre 2018.

Enviar correspondencia a: M. Patró-Hernández. Departamento de Personalidad, Evaluación y Tratamiento Psicológicos, Facultad de Psicología (Desp3.34). Campus de Espinardo. Universidad de Murcia. 30100, Murcia. España.

E-mail: rosapatro@um.es. 
S egún el Observatorio Español de las Drogas y las Adicciones (2017), el consumo de alcohol está más extendido entre los hombres que entre las mujeres, aunque tal diferencia parece ir disminuyendo en los últimos años. La Encuesta nacional sobre alcohol y otras drogas en España afirma que en el año 2015 el consumo de alcohol fue de un $82,9 \%$ entre los hombres, reduciéndose este porcentaje 10,8 puntos en las mujeres, con un 72,1\%. Esta diferencia entre ambos sexos ha sido mayor en las encuestas anteriores a 2013, siendo de 14,8 puntos en 2005 (84\% frente a $69,2 \%)$ y de 21,3 puntos en $1995(79,3 \%$ frente a $58 \%)$.

Distintos estudios han señalado importantes diferencias entre sexos respecto a la prevalencia del consumo de alcohol y el consumo problemático de alcohol. La prevalencia de problemas con el alcohol aumenta con el nivel de consumo de alcohol, teniendo las mujeres mayor probabilidad que los hombres de tener problemas con la bebida a igual patrón de consumo y, especialmente, bajo consumo severo (Bríñez-Horta, 2001; Ely, Hardy, Longford y Wadsworth, 1999). Las mujeres presentan un inicio del consumo más tardío y consumen menos cantidad, pero presentan una evolución hacia el abuso más rápida que los hombres, lo que se conoce como efecto telescoping, así como más dificultades para controlar el consumo de alcohol, más problemas asociados al consumo y mayor incidencia de patología depresiva y ansiosa (Alvanzo et al, 2011; Ávila y González, 2007; Ehlers et al., 2010; Díaz-Mesa et al., 2016; Míguez y Permuy, 2017; Sánchez-Autet et al., 2018). Estas diferencias son de gran interés para la práctica clínica, ya que hombres y mujeres presentan distintas formas de enfermar, distinto perfil, curso y pronóstico respecto a los problemas derivados del consumo de alcohol, lo que supone la necesidad de profundizar en el estudio de los factores determinantes de tales diferencias con el objetivo de mejorar el desarrollo de estrategias y programas de prevención sobre el consumo de alcohol.

En el campo de la salud, el estudio de las diferencias entre hombres y mujeres puede relacionarse con la conocida paradoja morbilidad-mortalidad (Nathanson, 1975; Verbrugge, 1982). Según la Organización Mundial de la Salud (2016), aunque los hombres tienen una esperanza de vida inferior a la de las mujeres, las mujeres sufren de peor salud y más enfermedades crónicas. Entre los factores que han sido señalados con el objetivo de explicar estas diferencias entre hombres y mujeres se encuentran: 1) riesgos biológicos; 2) riesgos adquiridos a través del trabajo y del estilo de vida; 3) las conductas que adoptan las personas para mejorar o para conservar la salud; 4) las formas utilizadas para comunicar los síntomas y asuntos de salud; y 5 ) el uso del sistema sanitario (Verbrugge, 1989).

El comportamiento humano es parcialmente influido por factores biológicos y factores ambientales. Estudios meta-analíticos basados en estudios de gemelos sugieren que los factores genéticos explican el 47\%, 46\% y $50 \%$ de la varianza observada en los aspectos cognitivos, los tras- tornos psiquiátricos y los trastornos neurológicos, respectivamente (Polderman et al., 2015), así como el 49\% de las diferencias observadas en trastornos por consumo de alcohol (Verhulst, Neale y Kendler, 2015). Aunque los factores asociados a los riesgos biológicos son importantes determinantes de la salud, el resto de factores, como los riesgos asociados al estilo de vida y las conductas de salud (por ejemplo, exposición a agentes tóxicos, microbianos, accidentes, incidentes con armas de fuego, uso ilícito de drogas, consumo de tabaco, alcohol, dieta inadecuada o falta de ejercicio), pueden llegar a ser igual de importantes, llegando a explicar, por ejemplo, el $50 \%$ de la mortalidad (Mokdad, Marks, Stroup y Gerberding, 2004). Por lo tanto, tener en cuenta las diferencias biológicas asociadas al sexo como única variable explicativa de la salud puede resultar ser reduccionista. En este sentido, la literatura defiende que ser hombre está asociado al aumento de la probabilidad de realizar conductas de riesgo para la salud (beber alcohol, fumar tabaco, no buscar ayuda médica, etc.), por lo que aumenta el riesgo de enfermar, lesionarse o de morir (Courtenay, 2000). A partir de ello, se ha desarrollado en el ámbito de la investigación un interés creciente por estudiar estos determinantes de la salud.

Teniendo en cuenta que el comportamiento humano está fuertemente regido por las normas sociales, algunos autores han planteado que, si el estilo de vida de los hombres les lleva a tener peor salud, resulta muy relevante averiguar por qué esas conductas de riesgo aparecen más en hombres y cómo promover conductas saludables, centrando su interés en el estudio de las diferencias comportamentales entre hombres y mujeres y, en concreto, respecto a la conformidad con las normas de género (Mahalik, Burns y Syzdek, 2007a).

Según Sánchez-López (2013), aunque en ocasiones se utilizan indistintamente los conceptos de sexo y género en la investigación sobre salud, ambos términos implican aspectos diferentes. El primero de ellos implica diferencias de corte más biológico, hormonales, cromosómicas, gonadales o dimorfismo cerebral y genital en relación a ser hombre o mujer, mientras que el segundo implica diferencias sociales y culturales respecto a los roles, relaciones, comportamientos, valores o actitudes que la sociedad atribuye diferencialmente a cada sexo, haciendo referencia a lo masculino y lo femenino. Algunas de las características asociadas con el rol tradicional masculino tienen que ver con un papel protector y proveedor de la familia y con rasgos tales como activo, decidido, competitivo, persistente y seguro de sí mismo, mientras que el rol tradicional femenino estaría relacionado con funciones de reproducción, crianza y soporte emocional de la familia y con rasgos como dedicación a los otros, emotividad, amabilidad, comprensión y calidez, entre otros (Sánchez-López, 2013; Sánchez-López y Limiñana-Gras, 2017). Después de que una persona entienda lo que la sociedad espera de ella, puede o no ajustarse a estas normas en función de una serie de 
variables contextuales e individuales, mostrando un mayor o menor grado de conformidad o aceptación de las mismas (Sánchez-López, Saavedra, Dresch y Limiñana-Gras, 2014). Las normas de género se adquieren a través de procesos de socialización y pueden mostrar algunas diferencias en función de los valores de cada cultura o sociedad, habiéndose encontrando, por ejemplo, diferencias en el grado de conformidad con ciertas normas de género masculinas entre muestras estadounidenses y españolas, mostrando los españoles menor conformidad con normas relacionadas con el desprecio hacia la homosexualidad, la importancia de ganar, la importancia del estatus, la atracción por la violencia y el riesgo, y mayor con la relacionada con el donjuanismo que los estadounidenses (Cuellar-Flores, Sánchez-López y Dresch, 2011). A partir de esta diferenciación entre los conceptos de sexo y género, revisiones recientes sobre la investigación en salud desde la perspectiva de género señalan la necesidad de tener en cuenta los factores sexo y género con el fin de profundizar en la comprensión de los determinantes de salud en ambos sexos, su interrelación y su implicación respecto a la exposición diferencial a factores de riesgo-protección y a resultados de salud y enfermedad diferentes (Sánchez-López y Limiñana-Gras, 2017).

El propósito de esta revisión es proporcionar evidencia sobre la investigación actual en el campo de estudio de las normas de género y su relación con el consumo de alcohol. Los objetivos de esta revisión son la exploración e identificación de estudios sobre género y consumo de alcohol, así como describir y analizar los resultados en función del instrumento utilizado. De esta manera, se pretende responder a la cuestión de si el género (y sus dimensiones) está relacionado con el consumo de bebidas alcohólicas en hombres y mujeres, y de qué modo.

\section{Método}

La revisión fue realizada siguiendo los pasos y etapas de identificación, tamización, elegibilidad e inclusión consignadas en la guía PRISMA ( preferred reporting items for systematic reviews and meta-analyses) (Liberati et al., 2009).

Para la identificación de los estudios, la búsqueda de artículos se llevó a cabo en tres bases de datos bibliográficas: PubMed, PsycINFO y ScienceDirect. Debido al carácter exploratorio de la revisión planteada no se establecieron criterios de restricción temporal para la búsqueda de estudios, incluyendo todos aquellos contenidos en las bases de datos hasta febrero de 2017. Se utilizaron los siguientes términos de búsqueda: "gender conformity", "conformity to masculine norms" "conformity to feminine norms" "masculinity", "feminity" en combinación con el término "alcohol" y los boleanos OR y AND. De forma complementaria, se realizó una búsqueda a través del portal Researchgate y a través de la revisión de las referencias utilizadas por los artículos que finalmente fueron seleccionados.

En la fase de tamización, tras un primer análisis de los artículos obtenidos, los criterios de inclusión aplicados fue-

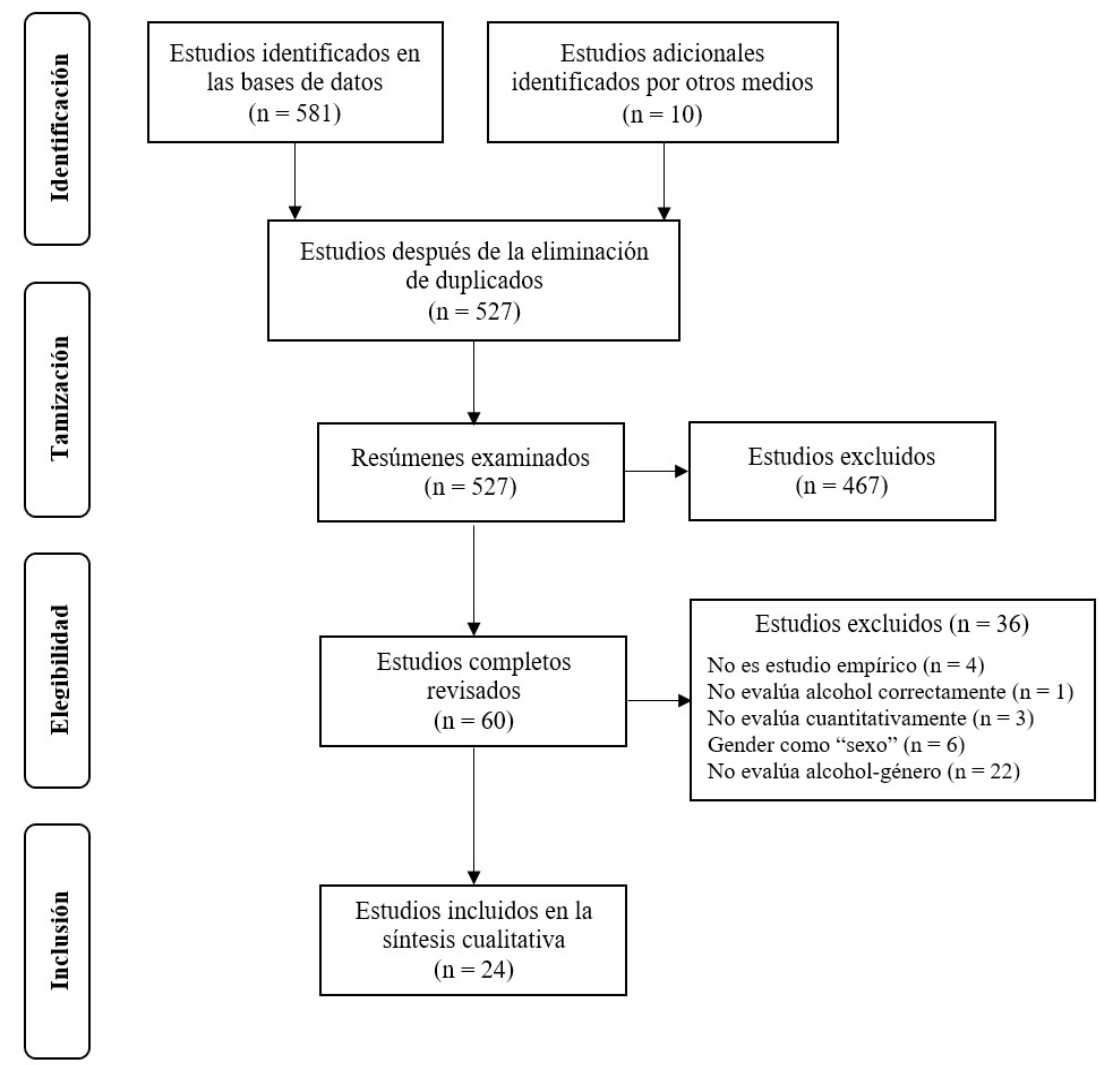

Figura 1. Diagrama de flujo del proceso de búsqueda y selección de estudios 
ron: a) inclusión de alguno de los términos de búsqueda en el título, resumen o palabras claves, b) materiales publicados en inglés o español, c) trabajos con acceso al texto completo y d) estudios empíricos, o trabajos que en base a la información contenida en el resumen no se pudiera determinar con exactitud si eran o no estudios empíricos. Dentro de la fase de elegibilidad, tras la lectura en profundidad del contenido de los artículos incluidos, se aplicaron los siguientes criterios de exclusión: a) no ser estudio empírico; b) estudios que no trataran acerca de la relación entre el género y el consumo o abuso del alcohol; c) "gender" en su traducción como "sexo" (hombre/mujer) en lugar de como "género" o "normas sociales" (masculino/femenino); d) alcohol no evaluado según el consumo o el abuso; e) resultados que no relacionen el consumo o abuso de alcohol con el género y sus factores; y f) evaluación realizada de forma cualitativa. Tras la aplicación de los criterios de exclusión, se redujo el número de artículos incluidos para la síntesis cualitativa a 24. La Figura 1 resume el número de trabajos incluidos en cada etapa del proceso de búsqueda y selección de estudios.

\section{Resultados}

La mayoría de estudios revisados están escritos en inglés, a excepción de dos (Brabete y Sánchez-López, 2012; Brabete, Sánchez-López, Cuéllar-Flores y Rivas-Diez, 2013). Se encontraron otros dos estudios llevados a cabo en España (Sánchez-López, Cuéllar-Flores y Dresch, 2012; Sánchez-López, Rivas-Diez y Cuéllar-Flores, 2013), dos en Suecia (Hensing y Spak, 2009; Hensing, Spak, Thundal y Östlund, 2003), cuatro en Australia (Mahalik, Levi-Minzi y Walker, 2007b; Ricciardelli, Williams y Kieman, 1998; Williams y Ricciardelli, 1999; Williams y Ricciardelli, 2001), uno en Alemania (Möller-Leimkühler, Schwarz, Burtscheidt y Gaebel, 2002) y los trece restantes en Estados Unidos.

En cuanto a muestras utilizadas, en algunos trabajos están compuestas únicamente de hombres (Good et al., 2008; Gordon et al., 2013; Iwamoto, Corbin, Lejuez y MacPherson, 2014; Iwamoto, Cheng, Lee, Takamatsu y Gordon, 2011), otros únicamente con mujeres (Kaya, Iwamoto, Grivel, Clinton y Brady, 2016), y el resto con muestra mixta.

La tabla 1 presenta los distintos instrumentos utilizados para evaluar la conformidad con las normas de género, desarrollados a lo largo de cuatro décadas, desde 1974 hasta 2014. La proporción de estudios por instrumento es muy discreta: el instrumento más utilizado es el CMNI (Mahalik et al., 2003), con un 37.5\%, seguido del CFNI (Mahalik et al., 2005), con $16.6 \%$. La utilización de cuestionarios con índices de fiabilidad por debajo de 0.80 , en todas o alguna de sus escalas, es relativamente frecuente, pero solo en dos de los 16 instrumentos se informa de índices de fiabilidad por debajo de 0.60 (ver en Kulis, Marsiglia, Lingard, Nieri y Nagoshi, 2008; y en Van Gundy, Schieman, Kelley y Rebellon, 2005). En otros casos, se han encontrado instrumentos con difícil acceso o menos conocidos, como es el caso del GEPAQ (Runge, Frey, Gollwitzer, Helmreich y Spence, 1981) o el MRNI-R (Levant et al., 2007). Respecto a la medida del consumo de alcohol, destacar que en la mayoría de los estudios suele incluirse a través de una serie de ítems en una batería de preguntas sobre la salud en general y en otros se utilizan cuestionarios específicos, como es el caso del Daily Drinking Questionnaire (DDQ) de Collins, Parks y Marlatt (1985); el Brief Young Adult Alcohol Consequences Questionnaire (B-YAACQ) de Kahler, Strong y Read (2005); o el Rutgers Alcohol Problem Index (RAPI) de White y Labouvie (1989).

En la Tabla 2 se exponen las principales características de los estudios incluidos en la revisión y sus principales resultados, que varían en función de los distintos instrumentos utilizados y las diferentes escalas incluidas en cada uno de ellos. En primer lugar, cabe mencionar tres estudios que no utilizan un instrumento estandarizado para evaluar la conformidad con normas de género, pero los ítems han sido seleccionados de otros estudios, investigaciones y/o instrumentos validados y estandarizados en el marco de otras investigaciones, para evaluar la identidad de género. El primero de ellos, el de Kulis et al. (2008), concluye que existe relación significativa entre Masculinidad Agresiva (agresividad, dominación, desobediencia) y consumo de sustancias, incluido el alcohol, así como entre Feminidad Afectiva (empatía, expresión de emociones) con un menor consumo de alcohol y con más conductas protectoras, todas ellas con un tamaño del efecto entre pequeño y moderado. En un estudio posterior (Kulis, Marsiglia y Nagoshi, 2010), se encontró una relación significativa entre Masculinidad Asertiva (asertividad, valor personal, confianza en uno mismo) y un menor consumo de alcohol en hombres, mientras que en mujeres la Masculinidad Agresiva y Feminidad Sumisa (sumisión, dependencia) se relacionó positivamente con un mayor consumo de alcohol, en todas con un tamaño del efecto moderado. Por ultimo, el estudio de Lye y Waldron (1998), en el que actitudes hacia roles de género no tradicionales se asociaron con un menor consumo de alcohol en los hombres, con un tamaño de moderado a grande; $y$ las actitudes no tradicionales hacia la convivencia y el matrimonio se asociaron a un menor consumo de alcohol tanto en hombres como en mujeres, particularmente para las actitudes hacia la convivencia, donde el tamaño del efecto es grande.

El resto de los estudios revisados utilizan instrumentos estandarizados, un total de 14 instrumentos incluyendo las versiones breves. A continuación, se describen los estudios que utilizaron instrumentos cuyo uso en el estudio del alcoholismo ha sido más discreto. El primero de ellos, el Personal Attributes Questionnaire (PAQ), utilizado por Van Gundy et al. (2005) en una muestra de hombres y mujeres de Toronto, quienes obtienen relaciones positivas entre Fe- 
Tabla 1. Instrumentos de medida de género utilizados en los estudios incluidos en la revisión

\begin{tabular}{|c|c|c|c|c|}
\hline & Instrumento & Autores/as y año & Propiedades psicométricas & Estudios en el que ha sido incluido \\
\hline 1 & $\begin{array}{l}\text { Bem Sex-Role Inventory } \\
\text { (BSRI) }\end{array}$ & Bem (1974) & $\begin{array}{l}60 \text { ítems. } 3 \text { escalas: Masculinidad, Feminidad y Deseabilidad } \\
\text { social. Consistencia interna entre } a=.70 \text { y } a=.86 \text {. }\end{array}$ & $\begin{array}{l}\text { Van Gundy, Schieman, Kelley y } \\
\text { Rebellon (2005) }\end{array}$ \\
\hline 2 & $\begin{array}{l}\text { Personal Attributes } \\
\text { Questionnaire (PAQ) }\end{array}$ & $\begin{array}{l}\text { Spence y Helmreich } \\
\text { (1978) }\end{array}$ & $\begin{array}{l}24 \text { ítems. } 2 \text { escalas: Instrumentalidad-Masculinidad y } \\
\text { Expresividad-Feminidad. Consistencia interna de } a=.69 \text { y } \\
a=.52 \text { respectivamente. }\end{array}$ & $\begin{array}{l}\text { Van Gundy, Schieman, Kelley y } \\
\text { Rebellon (2005) }\end{array}$ \\
\hline
\end{tabular}

3 Bem Sex-Role Inventory - Bem (1981) Short Form (BSRI-S)

4 Australian Sex-Role Scale Antill, Cunningham (ASRS) Russell y Thompson (1981)

5 German Extended Personal Attributes Questionnaire (GEPAQ)

6 Gender Role Conflict Scale (GRCS)

7 Masculine Role Norms Scale (MRNS)

8 Masculinity and Femininity Questionnaire (M/F-Q)

9 Cuestionario sobre roles de género y cohabitación

10 Conformity to Masculine Mahalik et al. (2003) Norms Instrument (CMNI)

Runge, Frey, Gollwitzer, 40 ítems. 2 escalas: Masculinidad y Feminidad.

Helmreich y Spence Consistencia interna de $a=.82$ y $a=.85$ respectivamente. (1981)

O’Neil, Helms, Gable, David y Wrightsman (1986)

Thompson y Pleck (1986)

Bergman et al. (1988)

Lye y Waldron (1998)

(1)

18 ítems. 3 escalas: Masculinidad-Personal, MasculinidadSocial y Feminidad. Consistencia interna entre $a=.66$ y $a=.92$.

40 ítems. 4 escalas: Masculinidad positiva, Masculinidad negativa, Feminidad positiva y Feminidad negativa. Consistencia interna entre $a=62$ y $a=81$

37 ítems. 4 factores: Éxito, Poder, Competición; Emocionalidad Good et al. (2008); Uy, Massoth y restrictiva; Restricción de los comportamientos afectivos entre Gottdiener (2014) hombres; Conflicto entre el trabajo y las relaciones familiares. Consistencia interna entre $\mathrm{a}=.80 \mathrm{y} \mathrm{a}=.87$.

26 ítems. 3 escalas: Estatus, Tenacidad y Antifeminidad. Consistencia interna entre $\mathrm{a}=.74 \mathrm{y} \mathrm{a}=.81$.

31 ítems. 4 escalas: Liderazgo, Preocupación por los demás, Auto-afirmación y Emotividad. Consistencia interna entre $a=.63$ y $a=.76$.

No estandarizado.

15 ítems seleccionados de cuatro cuestionarios distintos sobre actitudes tradicionales hacia roles de género. Consistencia interna de $a>60$.

94 ítems. 11 escalas: Ganar, Control emocional, Conducta Liu y Iwamoto (2007); Mahalik, de riesgo, Violencia, Poder sobre las mujeres, Dominancia, Levi-Minzi y Walkier (2007); Good, Donjuanismo, Independencia, Primacía en el trabajo, Desprecio hacia la homosexualidad y Búsqueda de posición social. Consistencia interna: Total $a=.94$; Escalas entre $\alpha=.72$ y $a=.91$.

Adaptación española (Cuéllar-Flores, Sánchez-López y Dresch, 2011). Total $a=.89$; Subescalas entre $a=.64$ y $\mathrm{a}=.81$.

Schopp, Thomson, Hathaway, Mazurek y Sanford-Martens (2008); Iwamoto et al. (2011); Iwamoto y Smiler (2013);

Brabete y Sánchez-López (2012);

Sánchez-López, Cuéllar-Flores y Dresch (2012); Brabete, SánchezLópez, Cuéllar-Flores y Rivas-Diez (2013); Sánchez-López, Rivas, Diez, Cuéllar-Flores (2013)

11 Conformity to Feminine Norms Instrument (CFNI)

Mahalik et al. (2005)

Cuidadora de niños, Delgadez, Fidelidad sexual, Modestia, Ser romántica en las relaciones, Hogareña e Invertir en la imagen o apariencia. Consistencia interna: Total $\mathrm{a}=.88$; Subescalas entre $a=.77$ y $a=.92$.

Adaptación española (Sánchez-López, Cuéllar-Flores, Dresch y Aparicio-García, 2009).Total a=.87; Subescalas entre $\mathrm{a}=.64 \mathrm{y} \mathrm{a}=.86$.

12 Male Role Norms Inventory-Revised (MRNI-R)

13 Cuestionario sobre identidad de género

14 Conformity to Masculine Norms Inventory-46 (CMNI-46)

15 Conformity to Feminine Norms Inventory-45 (CFNI-45)

16 Conformity to Masculine Norms Inventory-29 (CMNI-29)
Kulis, Marsiglia, Lingard, Nieri y Nagoshi (2008)

Levant et al. (2007) 53 ítems. 7 escalas: Evitación de la feminidad, Negatividad Uy, Massoth y Gottdiener (2014) hacia minorías sexuales, Autosuficiencia, Agresión, Dominancia, Sexualidad no relacional, Emocionalidad restrictiva. Consistencia interna: Total $a=.97$. Subescalas entre $a=.73$ y $a=.96$.

No estandarizado.

13 ítems derivados del Bem Sex-Role Inventory (BSRI) y Australian Sex-Role Scale (ASRS). 4 escalas: Masculinidad asertiva, Masculinidad negativa, Feminidad afectiva y Feminidad negativa. Consistencia interna entre $a=.50$ y $a=.66$.

Parent y Moradi (2009) Versión reducida del CMNI. 46 ítems. Consistencia interna subescalas entre $a=.77$ y $a=.91$. Corbin, Lejuez y MacPherson (2014)

Parent y Moradi (2010) Versión reducida del CFNI. 45 ítems. Se añade la escala Dulce y agradable. Consistencia interna subescalas entre $\mathrm{a}=.67$ y $\mathrm{a}=.90$.

Hsu y Iwamoto (2014) Versión reducida del CMNI. 29 ítems. 8 escalas: Ganar, Donjuanismo, Independencia, Violencia, Presentación heterosexual, Conducta de riesgo, Control emocional y Poder. Consistencia interna subescalas entre $a=.70$ y $a=.85$.
Iwamoto et al. (2011); Iwamoto,

Kulis, Marsiglia, Lingard, Nieri y Nagoshi (2010)

Kaya, Iwamoto, Grivel, Clinton y Brady (2016)

Kaya, Iwamoto, Grivel, Clinton y Brady (2016) Nagoshi (2008); Kulis, Marsiglia y 
minidad y consumo de alcohol en mujeres, con un tamaño moderado del efecto. El segundo, el German Extended Personal Attributes Questionnaire (GEPAQ), fue utilizado en un único estudio con muestra clínica (Möller-Leimkühler et al., 2002), en el que encontraron un predominio de identidad femenina y no diferenciada en mujeres y hombres alcohólicos, frente a un predominio de identidad masculina o andrógina en los hombres no alcohólicos, y de identidad andrógina en las mujeres no alcohólicas. En tercer lugar, la utilización del Gender Role Conflict Scale (GRCS) permitió señalar una relación directa, con un tamaño del efecto moderado, entre las dimensiones éxito, poder y competición de esta prueba y el número de bebidas alcohólicas por episodio (Good et al., 2008). Esta escala fue aplicada en otro estudio junto con el instrumento Male Role Norms Inventory Revised (MRNI-R) obteniendo resultados, con una magnitud del efecto grande, que relacionaban el consumo de alcohol en hombres con ideología tradicional masculina (Uy, Massoth y Gottdiener, 2014). En la misma línea, pero con un tamaño del efecto pequeño, y utilizando el instrumento Masculine Role Norms Scale (MRNS), se relacionaron ciertos aspectos de la masculinidad tradicional como, por ejemplo, dureza o "toughness" con el consumo de alcohol (Gordon et al., 2013).

Otros instrumentos han recibido una mayor atención por parte de los investigadores en el estudio del consumo de alcohol y otras sustancias. Este es el caso del Bem Sex Role Inventory (BSRI) y su forma breve (BSRI-S). En el estudio de Van Gundy et al. (2005), utilizando el BSRI, se encuentra una asociación negativa entre la masculinidad y el nivel de consumo de alcohol en los hombres; por otro lado, Vaughan, Wong y Middendorf (2014) utilizando el BSRI-S, que distingue entre una faceta personal y otra social de la masculinidad, encuentran que la masculinidad social se relaciona con el consumo excesivo de alcohol, tanto en hombres como en mujeres. Ambos estudios con tamaños de efecto grandes. De los estudios que utilizaron la Australian Sex Role Scale (ASRS) (Ricciardelli et al., 1998; Williams y Ricciardelli, 1999; Williams y Ricciardelli, 2001) se desprende que altas puntuaciones en las escalas de Masculinidad Negativa (agresividad, fanfarronería) estuvieron asociadas en ambos sexos a problemas con el alcohol (dependencia) y a conductas peligrosas derivadas del consumo (desinhibición), así como que bajas puntuaciones en Masculinidad y Feminidad Positiva (características asociadas positivamente con masculinidad, como asertividad y confianza en uno mismo, y con feminidad, como el amor por los hijos) se asociaron a personas con alta probabilidad de desarrollar problemas con el alcohol, mostrando estas relaciones un tamaño del efecto entre moderado y grande. Dos de los estudios revisados (Hensing et al., 2003; Hensing y Spak, 2009) utilizaron el Masculinity and Feminity Questionnaire (M/F-Q) con muestra exclusivamente femenina. Los resultados mostraron que las puntuaciones bajas en Liderazgo y Autoafirmación se relacionaban de forma significativa, aunque con un tamaño del efecto pequeño, con un aumento de la probabilidad de abuso y problemas con el alcohol. Por otro lado, y con un tamaño del efecto moderado, se hallaron relaciones significativas entre el aumento de esta probabilidad y puntuaciones altas en Emotividad.

Finalmente, se puede destacar un total de diez estudios que utilizan los instrumentos Conformity to Masculine Norms Inventory (CMNI) y Conformity to Feminine Norms Inventory (CFNI) y sus distintas versiones. Cuatro de los estudios revisados fueron realizados con la adaptación española del instrumento. Entre sus resultados, se encuentran relaciones de sentido positivo, con un tamaño del efecto moderado, entre el consumo de alcohol en hombres y la puntuación total de conformidad con normas masculinas, como con las escalas de Playboy (o Donjuanismo) y Búsqueda de posición social (Brabete y Sánchez-López, 2012). Con un tamaño del efecto pequeño, también con las normas de Dominancia, Violencia y Conductas de riesgo (Brabete et al., 2013; Sánchez-López et al., 2012), así como relaciones en sentido negativo con las escalas Control emocional y Declaración/presentación heterosexual (Brabete y Sánchez-López, 2012; Sánchez-López et al., 2012; Sánchez-López et al., 2013). En las mujeres, el consumo estuvo inversamente asociado a conformidad total con normas femeninas tradicionales (Brabete y Sánchez-López, 2012) y a las escalas Cuidado de niños (Brabete y Sánchez-López, 2012; Brabete et al., 2013), Fidelidad sexual (Brabete y Sánchez-López, 2012; Brabete et al., 2013; Sánchez-López et al., 2012; Sánchez-López et al., 2013), Hogareña y Modestia (Brabete et al., 2013), y Romanticismo en las relaciones (Brabete et al., 2013; Sánchez-López et al., 2012); solo la conformidad con la norma Invertir en la imagen o apariencia estuvo directamente relacionada con mayor consumo. El tamaño del efecto encontrado en la mayor parte de estas relaciones fue moderado.

Kaya et al. (2016), utilizaron muestra únicamente femenina aplicando las versiones cortas de los cuestionarios de conformidad con normas de género masculinas y femeninas (CMNI-29 y CFNI-45). Los resultados obtenidos indicaron asociaciones directas entre el consumo de alcohol y las escalas de Conductas de riesgo, Control emocional y Valorar agradablemente las relaciones, y asociaciones negativas o inversas con las escalas de Poder sobre las mujeres, Modestia y Fidelidad sexual. Respecto a los problemas derivados del consumo, las relaciones fueron directas para las escalas Conductas de riesgo, Delgadez e Invertir en la imagen o apariencia y negativas con la de Fidelidad sexual.

Por último, cabe destacar seis estudios que utilizan solo el CMNI exclusivamente con muestras de hombres, junto al estudio de Iwamoto y Smiler (2013) que lo hace con muestras de hombres y mujeres. Good et al. (2008), en el mismo estudio mencionado anteriormente, encuen- 
Tabla 2. Características y principales resultados de los estudios incluidos en la revisión

\begin{tabular}{|c|c|c|c|c|}
\hline $\begin{array}{l}\text { Autores y fecha de } \\
\text { publicación }\end{array}$ & País & Objetivo & Muestra e instrumentos & Principales resultados \\
\hline $\begin{array}{l}\text { Brabete y } \\
\text { Sánchez-López } \\
\text { (2012) }\end{array}$ & España & $\begin{array}{l}\text { Determinar si las normas de } \\
\text { género se relacionan con las } \\
\text { variables de salud en una } \\
\text { muestra de rumanos viviendo } \\
\text { en España. }\end{array}$ & $\begin{array}{l}188 \text { rumanos en España ( } 70 \text { mujeres / } 48 \\
\text { hombres) } \\
\text { Género: aCMNI y CFNI. } \\
\text { Alcohol: } 1 \text { pregunta sobre consumo } \\
\text { dentro de un test de salud. }\end{array}$ & 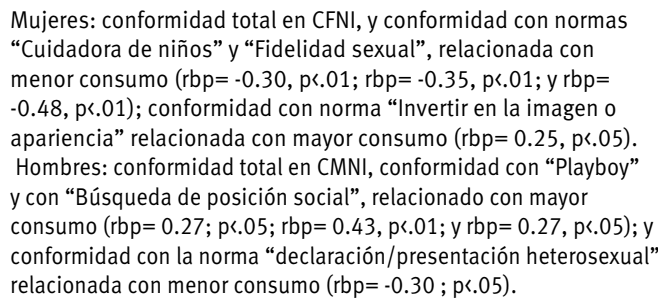 \\
\hline
\end{tabular}

Brabete et al. España Determinar si las normas de 750 rumanos en España (489 mujeres / Mujeres: conformidad total en CFNI, y conformidad con las normas

(2013) género se relacionan con el

261 hombres)

consumo de tabaco y alcohol Género: CMNI y CFNI.

en una muestra de rumanos Alcohol: dos preguntas para confirmar viviendo en España.
"Cuidadoras de niños", "Fidelidad sexual”, "Modestia”, "Ser

romántica en las relaciones" y "Hogareña”, relacionadas con

menor consumo $(r b p=0-.20, p<0.5 ; r b p=-0.19, p<0.5 ; r b p=-0.22$ $p<0.5 ; r b p=-0.20, p<0.5 ; y r b p=-0.11, p<0.5)$

Hombres: conformidad con normas "Conducta de riesgo",

"Violencia" y "Playboy", relacionada con mayor consumo $(\mathrm{rbp}=$ $0.11, \mathrm{p}<0.1 ; \mathrm{rbp}=0.11, \mathrm{p}<0.1 ; \mathrm{y} r \mathrm{rp}=0.10, \mathrm{p}<0.1)$. los roles masculinos y los conflictos asociados al consur de alcohol en hombres con lesiones graves.

52 hombres con lesiones de médula espinal y traumatismo cerebral.

Género: bGRCS y CMNI.

se asocian a mayor consumo ( $r s=0.46, p<.05)$

Mayor conformidad con la norma "Dominancia", relacionado con y la frecuencia actual de binge drinking. consumo abusivo o binge drinking ( $r s=0.47, p<.05$ ).

Gordon et al. (2013) EEUU Examinar la relación entre las 296 hombres.

normas masculinas tradicionales Género: cMRNS.

de una diversidad étnica y racial Alcohol: pregunta sobre consumo de de hombres jóvenes que han

pasado a ser padres y el consum

de sustancias (tabaco, alcohol,

marihuana, drogas) y sus hábitos

saludables (dieta, ejercicio).

Hensing y Spak Suecia Analizar en mujeres la asociación 930 mujeres.

entre

Género: dM/F-Q

de identidad de género y las

Alcohol: AUD (abuso y dependencia) y

variables de consumo de alcohol HED (al menos $60 \mathrm{~g}$ de etanol en un solo

(1AUD y $1 \mathrm{HED})$

día al menos una vez al mes).

Hensing et al. (2003) Suecia Analizar las dimensiones de 836 mujeres.

la identidad de género y su Género: $M / F-Q$.

asociación con trastornos

psiquiátricos y el consumo de

alcohol (1HED y $3 \mathrm{HAC}$.

Alcohol: Dependencia y abuso (DSM-III-R),

HED (al menos $60 \mathrm{~g}$ de etanol en un solo día al menos una vez al mes) y HAC (al menos $\geq 600$ g etanol por mes durante los últimos 12 meses).

Iwamoto y Smiler EEUU Analizar la relación entre la

(2013) conformidad de género, la de alcohol en población joven. " "cerveza, refrescos de vino o sidra".

Iwamoto et al.

EEUU

Analizar la relación entre el género y los factores de riesgo de intoxicación y otros problemas relacionados con el alcohol, específicamente en

\section{6 hombres.}

Género: eCMNI-46.

Alcohol: Beber hasta la intoxicación y

Problemas Relacionados con el Alcohol

(4 RAPI).

Mujeres con bajo "Liderazgo", mayor probabilidad de abuso y dependencia del alcohol [OR $(95 \% \mathrm{Cl})=1.95(1.17-3.26)]$.

Mayor respaldo a la norma de dureza o "Toughness" se relaciona con mayor consumo de alcohol [OR $(95 \% \mathrm{Cl})=1.725$; p .01]. La norma "Estatus" se relaciona, aunque de forma muy débil, con menor consumo [OR $(95 \% \mathrm{Cl})=0.78 ; \mathrm{p}=.063]$.

Mujeres con bajo "Liderazgo", mayor probabilidad de dependencia y abuso $[\mathrm{OR}(95 \% \mathrm{Cl})=1.93(1.23-3.01)]$. Mujeres con baja "Auto-afirmación" mayor probabilidad de dependencia y abuso [OR $(95 \% \mathrm{Cl})=1.98(1.25-3.07)]$ Mujeres con alta "Emotividad", mayor probabilidad de dependencia y abuso [OR $(95 \%)=3.22(1.96-5.30)]$

La mayor conformidad con las normas de "declaración/presentación heterosexual" (16, pr.05), Playboy $(.25, p<.01)$, y "conductas de riesgo" $(.17, p<01)$ predijo el consumo de alcohol en varones. La mayor conformidad con la norma "conductas de riesgo" (.20, pr.01) predijo el consumo de alcohol en mujeres.

Las normas masculinas de "ganar" $(r=0.14, p<.01)$, "conductas de riesgo" $(r=0.20, \mathrm{p}<, 01)$, y "playboy” $(r=0.25, p<.01)$, fueron positivamente relacionados con beber hasta la intoxicación. Las normas masculinas asunción de "conductas de riesgo" $(r=$ $0.19, p<.01)$, "poder sobre las mujeres" $(r=0.20, p<.01)$, playboy Género: 5 de las subescalas del CMNI. presión de grupo y el consumo Alcohol: frecuencia con la que se consumen $(r=0.28, p<.01)$, “independencia” ( $r=0.10, p<.01)$, y “primacía de trabajo" ( $r=0.09, p$ <.05) se asociaron con un aumento de los problemas relacionados con el alcohol.

Las tres normas masculinas que aumentan el riesgo de problemas relacionados con el alcohol son "playboy" (IRR $=5,01, p<0.001)$, "conductas de riesgo" (IRR $=2,66, p<0.001)$, e "independencia" $(\mathrm{IRR}=3,12, p<0.001)$

Las normas masculinas relacionadas directamente con mayor consumo son "Playboy" $(r=0.26, p<0.1)$, "Conducta de riesgo" $(r=$ $0.1, p<0.1)$ y "Ganar" ( $r=0.23$ ( $p<0.1)$

expectativas positivas de Género: CMNI-46. consumo de alcohol como Alcohol: 5DDQ (Estimación del consumo mediador entre normas de género de alcohol en los últimos tres meses) y un masculinas y consumo de alcohol ítem sobre binge drinking. entre hombres universitarios.

Kaya et al. (2016) EEUU Examinar la conformidad 645 mujeres.

de las mujeres a las normas masculinas y femeninas y su relación con el consumo y la presencia de problemas relacionados con alcohol en una 645 muje $\mathrm{CCMNI}-29$, gCFNI-45.

Alcohol: HED (bebida episódica intensa) y problemas relacionados con el alcohol (6B-YAACQ) muestra de universitarias.
La norma masculinas de "presentación heterosexual" está inversamente relacionada con la frecuencia en el consumo de alcohol $(r=-0.13, p<0.1)$

Tres normas masculinas se asociaron significativamente a HED:

"conductas de riesgo" y "control emocional" con mayor consumo $(\operatorname{IRR}=1.11, p<.05, y \operatorname{IRR}=1.07, \mathrm{p}<.05) ; \mathrm{y}$ "poder sobre las mujeres" con menor consumo (IRR $=.89, p<.01)$

Las normas femeninas significativamente asociadas con HED fueron tres: "modestia" (IRR=.93, p <.05) y "fidelidad sexual" 
(IRR=.86, $\mathrm{p}<.001)$ con una disminución de HED, "relacional" (IRR=1.06, p «.05) con mayor HED.

La norma masculina de "conductas de riesgo" $(I R R=1.65, p$ <.001), y las normas femeninas de "delgadez" (IRR $=1.20, p$ <.01) e "invertir en apariencia" (IRR=1.16, $p<.01)$, se asociaron positivamente con los problemas relacionados con el alcohol. La norma femenina de "fidelidad sexual" se asoció negativamente con problemas relacionados con el alcohol (IRR=.70, p <.001).

\begin{tabular}{|c|c|c|c|}
\hline Kulis et al. (2010) & EEUU & $\begin{array}{l}\text { Analizar la relación entre roles } \\
\text { de género positivos y negativos, } \\
\text { problemas de comportamiento } \\
\text { externalizantes e internalizantes } \\
\text { y consumo de sustancias. }\end{array}$ & $\begin{array}{l}151 \text { estudiantes latinos. } 91 \text { mujeres, } 60 \\
\text { hombres. } \\
\text { Género: medida de } 12 \text { ítems para cuatro } \\
\text { orientaciones de roles de género. } \\
\text { Alcohol: } 6 \text { ítems sobre consumo. }\end{array}$ \\
\hline Kulis et al. (2008) & EEUU & $\begin{array}{l}\text { Analizar si las medidas de } \\
\text { identidad de género predicen } \\
\text { el consumo de sustancias, } \\
\text { intenciones de consumo, } \\
\text { expectativas, entre otras. }\end{array}$ & $\begin{array}{l}327 \text { estudiantes latinos. } \\
\text { Género: medida de } 12 \text { ítems para cuatro } \\
\text { orientaciones de roles de género. } \\
\text { Alcohol: } 6 \text { ítems sobre consumo. }\end{array}$ \\
\hline
\end{tabular}

"Masculinidad Asertiva" relacionada con menor consumo de alcohol en los hombres ( $r=-0.27, p<.05)$.

"Masculinidad Agresiva” y “Feminidad Sumisa”, relacionada con mayor consumo en las mujeres $(r=0.31, p<.01 ; r=0.25$, pr.05)

"Masculinidad agresiva" se asoció con mayor consumo de sustancias, incluido el alcohol (puntuaciones de $r=0.12$ a 0.29 ). "Feminidad afectiva" se asoció con menor uso reciente de alcohol $(r=-0.21)$ y menos consumo excesivo de alcohol $(r=-0.17)$, con más conductas protectoras, por ej. la menor intención de consumo $(r=-0.12)$, y la menor aprobación $(r=-0.28)$.

\begin{tabular}{llll}
\hline Liu y lwamoto & EEUU & Explorar la relación entre valores & 154 estudiantes asiático-americanos. \\
& asiáticos, roles de género, & Género: CMNI. \\
& estilos de afrontamiento y & Alcohol: Consumo de alcohol y binge \\
& consumo de sustancias. & drinking behavior.
\end{tabular}

Mayor consumo de alcohol estuvo relacionado positivamente a conformidad total con normas masculinas $(r=0.17$, pr.05), Ganar $(r=0.20$, pr.05), búsqueda de status $(r=29, p<.01)$, Playboy $(r=20$ $p<.05)$, Conducta de riesgo ( $r=17$, p<.05) y violencia $(r=17$, p<.05); y negativamente con control emocional $(r=-0.20$, pr.05). Binge drinking behavior estuvo asociado a conformidad total con normas masculinas ( $r=0.18$, pr.05), y a las normas de "Ganar" ( $r=19$, pr0.5), "búsqueda de status" ( $r=23, p<.01)$, y "Playboy" $(r=29$, pr.01); y negativamente con control emocional ( $r=-0.18$, p<.05). La norma "Poder sobre las mujeres" predijo significativamente el abuso de alcohol (OR=1.31, p<.01)

\begin{tabular}{|c|c|c|c|}
\hline $\begin{array}{l}\text { Lye y Waldron } \\
\text { (1998) }\end{array}$ & EEUU & $\begin{array}{l}\text { Analizar la relación entre } \\
\text { consumo de alcohol, de } \\
\text { marihuana y otras drogas } \\
\text { ilegales y las actitudes acerca } \\
\text { de los roles de género, la familia } \\
\text { y la cohabitación. }\end{array}$ & $\begin{array}{l}\text { El rango de muestra varía entre } 756-963 \\
\text { mujeres y } 821-1095 \text { hombres. } \\
\text { Género: } 36 \text { ítems de cuatro cuestionarios } \\
\text { distintos sobre actitudes no tradicionales } \\
\text { hacia roles de género, y hacia la } \\
\text { convivencia y el matrimonio. } \\
\text { Alcohol: ítems acerca del consumo } \\
\text { pasado y en los últimos } 30 \text { días. }\end{array}$ \\
\hline
\end{tabular}

Las actitudes hacia roles de género no tradicionales en los hombres se asociaron con un menor consumo de alcohol (p<.001). Entre las mujeres, solo hubo unas pocas relaciones y más débiles e inconsistentes entre las medidas de os roles de género tradicionales y el consumo de alcohol.

Las actitudes no tradicionales hacia la cohabitación y el matrimonio se asociaron fuertemente a un menor consumo de alcohol tanto en hombres como en mujeres, particularmente para las actitudes hacia la convivencia. (p<.001).

\begin{tabular}{|c|c|c|}
\hline $\begin{array}{l}\text { Mahalik, Levi-Minzi } \\
\text { y Walker (2007b) }\end{array}$ & Australia & $\begin{array}{l}\text { Confirmar si la salud de los } \\
\text { hombres australianos y sus } \\
\text { conductas saludables tienen } \\
\text { una relación significativa con } \\
\text { la conformidad de normas } \\
\text { tradicionales masculinas. }\end{array}$ \\
\hline
\end{tabular}

253 hombres australianos.

Género: CMNI.

Alcohol: ítem dentro del cuestionario de

La frecuencia de conductas de riesgo para la salud (incluido el consumo de alcohol) se asoció con las normas "Independencia" $(r=0.24$, pr.001), "Donjuanismo" ( $r=0.29$, pr.001) y "Violencia" $(r=0.26, p<.001)$

Möller-Leimkühler Alemania Explorar si el acercamiento et al. (2002) a la orientación de género sería útil en la contribución de la hipótesis del aumento de mujeres con alcoholismo debido al cambio de los roles femeninos tradicionales.

salud Health Behavior Inventory ( $\mathrm{HBI}$ ).

112 personas actualmente desintoxicadas Diferencias significativas para cada una de las cuatro identidades (36 mujeres, 76 hombres). Género: Orientación del rol de género (hGEPAQ).

Alcohol: resultados de una evaluación en roles de género en ambas muestras, la de alcohólicos y no alcohólicos (pr.05):

- predominio en mujeres y hombres alcohólicos de identidad femenina y no diferenciada;

- predominio en los hombres no alcohólicos de identidad masculina 0 andrógina; - predominio en las mujeres no alcohólicas de identidad andrógina.

\begin{tabular}{|c|c|c|c|}
\hline $\begin{array}{l}\text { Ricciardelli et al. } \\
\text { (1998) }\end{array}$ & Australia & $\begin{array}{l}\text { Investigar la relación entre } \\
\text { aspectos deseables y no } \\
\text { deseables de la masculinidad y } \\
\text { feminidad y aspectos derivados } \\
\text { de comer (restricción) y beber } \\
\text { bebidas alcohólicas. }\end{array}$ & $\begin{array}{l}114 \text { universitarias. } \\
\text { Género: iASRS. } \\
\text { Alcohol: dos ítems sobre consumo } \\
\text { a la semana y cuestionario Alcohol } \\
\text { Dependence Scale. }\end{array}$ \\
\hline $\begin{array}{l}\text { Sánchez-López } \\
\text { et al. (2012) }\end{array}$ & España & $\begin{array}{l}\text { Evaluar si el género está } \\
\text { relacionado con el consumo de } \\
\text { sustancias y las enfermedades } \\
\text { crónicas. }\end{array}$ & $\begin{array}{l}\text { Universitarios españoles. } 234 \text { mujeres y } \\
226 \text { hombres. } \\
\text { Género: CMNI y CFNI. } \\
\text { Alcohol: ítem sobre frecuencia de } \\
\text { consumo de alcohol. }\end{array}$ \\
\hline
\end{tabular}

"Negative Masculinity" se asoció a altas puntuaciones en “Dependencia del Alcohol” y “Desinhibición” (p<.05).

Consumo en hombres estuvo directamente asociado a conformidad con normas de "Dominancia" $(r=0.138$, pr.05) y "Playboy" ( $r=0 . .199, p<.05)$

Consumo en mujeres estuvo inversamente asociado a conformidad total $(r=-0.244$, p <.001), "Fidelidad sexual" ( $r=$ $0.277, p<.001)$ y “Ser romántica” ( $r=-0.331$, p $<.001)$ La conformidad con las normas "Playboy" y "dominancia", en sentido positivo, y la de "ganar", en sentido negativo o inverso, explicaron el $7 \%$ de la varianza del consumo de alcohol en hombres ( $p<.001)$. La conformidad con las normas femeninas "ser romántica" y "fidelidad sexual "se asociaron con un menor consumo de alcohol explicando el $13 \%$ de la varianza en mujeres ( $F=19.61 ; p<.001)$

\begin{tabular}{|c|c|c|c|c|}
\hline $\begin{array}{l}\text { Sánchez-López et } \\
\text { al. (2013) }\end{array}$ & España & $\begin{array}{l}\text { Analizar el impacto de la } \\
\text { conformidad con las normas de } \\
\text { género en el consumo de tabaco } \\
\text { y alcohol. }\end{array}$ & $\begin{array}{l}\text { Universitarios españoles. } 435 \text { mujeres y } \\
419 \text { hombres. } \\
\text { Género: CMNI y CFNI. } \\
\text { Alcohol: ítems sobre frecuencia de } \\
\text { consumo en las últimas dos semanas } \\
\text { extraídos de la encuesta Nacional de } \\
\text { Salud (INE, 2006). }\end{array}$ & $\begin{array}{l}\text { Los hombres con puntajes más altos en la escala de "control } \\
\text { emocional" fueron menos propensos a consumir alcohol } \\
\text { (R2=7.6\%, p <.001). } \\
\text { Los hombres con puntajes más altos en la escala de "playboy y } \\
\text { "violencia" fueron más propensos a consumir alcohol ( } R 2=10,2 \% \\
\text { y } 7.4 \% \text {, p } 2.01 \text { y p } .05 \text { respectivamente) } \\
\text { Las mujeres con puntajes más altos en la escala de "fidelidad sexual" } \\
\text { fueron menos propensas a consumir alcohol ( } R 2=10,4 \% \text {, } p<.01) \text {. }\end{array}$ \\
\hline
\end{tabular}




\begin{tabular}{|c|c|c|c|c|}
\hline Uy et al. (2014) & EEUU & $\begin{array}{l}\text { Analizar el vínculo entre la } \\
\text { ideología tradicional masculina, } \\
\text { el conflicto de roles de género, } \\
\text { los motivos para consumir y el } \\
\text { consumo de alcohol. }\end{array}$ & $\begin{array}{l}109 \text { hombres. } \\
\text { Género: jMRNI-R y kGRCS. } \\
\text { Alcohol: Drinking Motives Questionnaire- } \\
\text { Revised y Daily Drinking Questionnaire- } \\
\text { Revised. }\end{array}$ & $\begin{array}{l}\text { Se observó un efecto indirecto total significativo de las ideologías } \\
\text { masculinas tradicionales en los problemas con la bebida (.19, } \\
\text { p«.05) } \\
\text { El } 25.9 \% \text { de la varianza asociada con el consumo de alcohol se } \\
\text { debió a ideologías masculinas tradicionales y motivos para el } \\
\text { consumo de alcohol. }\end{array}$ \\
\hline $\begin{array}{l}\text { Van Gundy et al. } \\
\text { (2005) }\end{array}$ & EEUU & $\begin{array}{l}\text { Examinar los efectos del sexo y } \\
\text { de la masculinidad y feminidad } \\
\text { en el consumo de alcohol. }\end{array}$ & $\begin{array}{l}\text { Moscú: muestra de 1996, } 804 \text { personas. } \\
\text { // } \\
\text { Toronto: muestra de 1995, } 1361 \\
\text { personas. } \\
\text { Género: IBSRI (Moscú) y mPAQ (Toronto). } \\
\text { Alcohol: frecuencia de consumo en } \\
\text { últimos } 30 \text { días y cantidad (Moscú) y } \\
\text { frecuencia últimos } 12 \text { meses y cantidad } \\
\text { (Toronto). }\end{array}$ & $\begin{array}{l}\text { Interacción significativa sexo*masculinidad en la muestra de } \\
\text { Moscú: la masculinidad se asocia negativamente con el nivel de } \\
\text { consumo de alcohol en los hombres, y de forma débil y positiva } \\
\text { entre las mujeres ( } b=.808 ; p<.01) \\
\text { Interacción significativa sexo*feminidad en la muestra de Toronto: } \\
\text { la feminidad aumenta el consumo de alcohol de las mujeres (b } \\
=.366 ; \text { p<.05) }\end{array}$ \\
\hline $\begin{array}{l}\text { Vaughan et al. } \\
\text { (2014) }\end{array}$ & EEUU & $\begin{array}{l}\text { Probar la relación entre roles de } \\
\text { género y binge drinking en una } \\
\text { muestra de latinos adultos. }\end{array}$ & $\begin{array}{l}660 \text { personas. } \\
\text { Género: nBSRI-S (feminidad, } \\
\text { masculinidad social y masculinidad } \\
\text { personal) } \\
\text { Alcohol: binge drinking y problemas de } \\
\text { alcohol. }\end{array}$ & $\begin{array}{l}\text { La Masculinidad social se asoció a un consumo excesivo de } \\
\text { alcohol en hombres y mujeres (OR= }>100 \text {, p<.05). }\end{array}$ \\
\hline $\begin{array}{l}\text { Williams y } \\
\text { Ricciardelli (1999) }\end{array}$ & Australia & $\begin{array}{l}\text { Examinar la relación entre } \\
\text { características estereotipadas } \\
\text { de género y consumo de } \\
\text { alcohol. }\end{array}$ & $\begin{array}{l}422 \text { universitarios ( } 243 \text { mujeres y } 179 \\
\text { hombres). } \\
\text { Género: ASRS. } \\
\text { Alcohol: cuestionario Short Form } \\
\text { Michigan Alcoholism Screening Test } \\
\text { (SMAST), promedio de bebidas por } \\
\text { episodio y promedio de días de bebida } \\
\text { por semana. }\end{array}$ & $\begin{array}{l}\text { Se identifica dos patrones de consumo: } \\
\text { Uno asociado a Masculinidad negativa (.69) y a Feminidad } \\
\text { positiva }(-.86) \text {. } \\
\text { Otro asociado a Masculinidad y Feminidad positiva }(-.96 \text { y }-.41) \\
\text { Se destaca la importancia de incluir atributos de género, tanto } \\
\text { positivos como negativos, en el estudio de los estereotipos de } \\
\text { género y las conductas de consumo de alcohol. }\end{array}$ \\
\hline $\begin{array}{l}\text { Williams y } \\
\text { Ricciardelli (2001) }\end{array}$ & Australia & $\begin{array}{l}\text { Estudiar la relación entre } \\
\text { los síntomas de problemas } \\
\text { de alcohol y desórdenes } \\
\text { alimenticios con las } \\
\text { características de género y con } \\
\text { las evidencias de comorbilidad } \\
\text { en estudiantes. }\end{array}$ & $\begin{array}{l}217 \text { mujeres universitarias. } \\
\text { Género: ASRS. } \\
\text { Alcohol: cuestionario SMAST, promedio } \\
\text { de bebidas por episodio y promedio de } \\
\text { días de bebida por semana y The Alcohol } \\
\text { Dependence Scale (ADS). }\end{array}$ & $\begin{array}{l}\text { Se identifica dos patrones de consumo: } \\
\text { Uno asociado a Masculinidad Positiva (.89) y Feminidad Negativa } \\
(-.61) \text {. } \\
\text { Otro a Masculinidad Negativa (.85). }\end{array}$ \\
\hline
\end{tabular}

Nota. Instrumentos de Género: $\mathrm{a}=$ Conformity to Masculine/Feminine Norms Inventory, $\mathrm{b}=$ Gender Role Conflict Scale, $\mathrm{c}=$ Masculine Role Norms Scale, $\mathrm{d}=$ Masculinity and Femininity Questionnaire, e = Conformity to Masculine Norms Inventory-46, $\mathrm{f}=$ Conformity to Masculine Norms Inventory-29, $\mathrm{g}=$ Conformity to Feminine Norms Inventory-45, $\mathrm{h}=$ German Extended Personal Attributes Questionnaire, $\mathrm{i}=$ Australian Sex-Role Scale, $\mathrm{j}=$ Male Role Norms Inventory-Revised, $\mathrm{k}=$ Gender-Role Conflict Scale, I = Bem Sex Role Inventory, $\mathrm{m}=$ Personal Attributes Questionnaire, $\mathrm{n}=$ Bem Sex Role Inventory-Short Form.

Notas:1AUD= Trastornos por consumo de alcohol: dependencia y abuso; $2 \mathrm{HED}=$ Heavy episodic drinking (bebida episódica intensa), $3 \mathrm{HAC}=\mathrm{High}$ alcohol consumption, 4RAPI= Rutgers Alcohol-Related Problems, $5 \mathrm{DDQ}=$ Daily Drinking Questionnaire, 6B-YAACQ= Brief Young Adult Alcohol Consequences Questionnaire.

tran asociaciones directas, también con una magnitud del efecto moderada, entre el consumo excesivo o de borrachera, y la norma masculina de Dominancia del CMNI. En el estudio de Iwamoto et al. (2011) la intoxicación etílica y los problemas relacionados con el alcohol se asociaron directamente con las escalas de Ganar, Conductas de riesgo, Playboy o Donjuanismo, Poder sobre las mujeres, Independencia y Primacía en el trabajo, todas con tamaños del efecto de pequeño a moderado. Asimismo, los resultados mostraron que las normas masculinas que aumentan el riesgo de problemas relacionados con el alcohol son la norma Playboy, con un tamaño moderado del efecto, y las normas Conductas de riesgo e Independencia, con un tamaño pequeño (Iwamoto et al., 2011). En el estudio de Iwamoto y Smiller (2013) la mayor conformidad con la norma Conductas de riesgo y Presentación heterosexual predijo el consumo de alcohol en varones, y la norma Conductas de riesgo en mujeres. En general, los resultados de los estudios de Iwamoto y colaboradores en las muestras de hombres mostraron una correlación positiva entre el consumo de alcohol y las escalas del CMNI de Playboy, Ganar y Conductas de riesgo, con tamaños del efecto de pequeño a moderado, así como una relación directa entre el au- mento del riesgo de problemas con el alcohol y las escalas Conductas de riesgo, Independencia y Playboy (Iwamoto y Smiler, 2013; Iwamoto et al., 2014; Liu y Iwamoto, 2007). Por último, el estudio de Mahalik et al., 2007b) encontró una relación directa entre el mayor número de conductas de riesgo para la salud (entre ellas el consumo elevado de alcohol) y las escalas Independencia, Donjuanismo y Violencia, con tamaños del efecto moderados.

\section{Discusión}

El objetivo de este trabajo era proporcionar evidencia, mediante una revisión sistemática, sobre la investigación que se ha realizado en el área de estudio de la conformidad con las normas de género respecto a su posible relación con el consumo de alcohol en hombres y mujeres, asî como sintetizar las principales conclusiones obtenidas en la investigación sobre la temática.

Uno de los principales aspectos observados en esta revisión ha sido la diversidad de conclusiones a las que han llegado los distintos autores. Esto podría ser explicado por la variabilidad de cuestionarios utilizados y las diferentes características y procedencias de las muestras objeto de es- 
tudio, siendo unos pocos resultados los que pueden llegar a ser generalizables. En general, y a pesar de esta diversidad, se pueden destacar tres aspectos sobre los que se han obtenido evidencias consistentes en los estudios revisados respecto a la relación entre género y consumo de alcohol.

En primer lugar, el mayor consumo de alcohol parece estar relacionado con la masculinidad. Distintos estudios han encontrado, con un tamaño del efecto grande, que la ideología tradicional masculina se relaciona con un mayor consumo de alcohol en hombres (Uy et al., 2014) y la faceta o dimensión social asociada a la masculinidad con el consumo excesivo o de borrachera (Vaughan, Wong y Middendorf, 2014). Igualmente son destacables, con magnitudes del efecto moderadas, algunas dimensiones concretas relacionadas con las normas de género asociadas al consumo de alcohol en hombres como la agresividad (Kulis et al., 2008; Mahalik et al., 2007b; Sánchez-López et al., 2013), la dominancia (Brabete y Sánchez-López, 2012; Good et al.,2008), las conductas de riesgo (Iwamoto et al., 2011; Iwamoto et al., 2014; Iwamoto y Smiler, 2013; Kaya et al., 2016), el donjuanismo (Brabete y Sánchez-López, 2012; Iwamoto et al., 2011; Iwamoto et al., 2014; Iwamoto y Smiler, 2013; Liu y Iwamoto, 2007; Mahalik et al., 2007b; Sánchez-López et al., 2012; Sánchez-López et al., 2013), la independencia (Mahalik et al., 2007b), el éxito (Good et al.,2008; Iwamoto y Smiler, 2013; Liu y Iwamoto, 2007) o la búsqueda de admiración o posición social (Brabete y Sánchez-López, 2012; Liu y Iwamoto, 2007).

En segundo lugar, parece existir de igual modo cierto consenso respecto a la relación entre consumo de alcohol y las normas de género femeninas. En general, las características que definen la feminidad tradicional aparecen como variables protectoras ante el consumo de alcohol. Tanto la conformidad general con las normas tradicionales femeninas, como algunas dimensiones concretas como el interés por la crianza de los hijos (Brabete y Sánchez-López, 2012; Brabete et al., 2013), la fidelidad sexual (Brabete y Sánchez-López, 2012; Brabete et al., 2013; Kaya et al., 2016; Sánchez-López et al., 2013), ser hogareña (Brabete y Sánchez-López, 2012; Brabete et al., 2013) o ser romántica en las relaciones (Brabete et al., 2013; Sánchez-López et al., 2012), aparecen como variables relacionadas con un menor consumo de alcohol en mujeres, con magnitudes del efecto de moderadas a grandes.

En tercer lugar, a pesar de disponer de cierta evidencia sobre la relación entre comportamientos tradicionalmente masculinos y el consumo de alcohol y sobre el perfil protector de aspectos asociados a la feminidad tradicional ante este consumo, no ocurre lo mismo a la inversa, es decir, no existe tanta evidencia acerca de variables protectoras en hombres, o acerca de las variables relacionadas con un mayor consumo en mujeres. Algunos trabajos confirman la hipótesis de que los hombres con mayor conformidad con aspectos relacionados con la masculinidad positiva o asertiva
(Gordon et al., 2013; Kulis et al., 2010), consumen menos alcohol, así como que las mujeres con mayor identificación con aspectos negativos relacionados con la feminidad tradicional, como la sumisión, y con la masculinidad, como la agresividad, consumen más (Kulis et al., 2010; Van Gundy et al., 2005). Sin embargo, son datos muy discretos que tendrían que ser estudiados con mayor profundidad y, dado el empleo de cuestionarios diseñados para hombres para evaluar a mujeres, con el desarrollo de instrumentos de medida que puedan evaluar tanto la identificación con normas masculinas como femeninas, adaptados para ambos sexos.

Por otro lado, a partir de la revisión realizada es posible plantear la cuestión de la relevancia de tener en cuenta la evolución de las normas y roles sociales. Algunos autores señalan que el rol tradicional de la mujer se está viendo transformado y con él las razones para beber. Por ejemplo, se sugiere que uno de los motivos para beber en las mujeres puede estar basado en la necesidad de sentirse igual que los hombres, a través de la adopción de comportamientos de riesgo para la salud, como el consumo de alcohol, en aquellas mujeres que muestran una mayor identificación o conformidad con aspectos negativos asociados a las normas de género tradicionales relacionadas con la masculinidad (Kaya et al., 2016; Williams y Ricciardelli, 1999). Así, se señala la necesidad de plantear nuevas hipótesis de investigación para estudiar la influencia que la conformidad con las normas de género, tanto masculinas como femeninas, pueden tener sobre las conductas de salud de las mujeres, dados los cambios de los roles de hombres y mujeres en la sociedad y la evidencia de un aumento en el consumo de bebidas alcohólicas en mujeres (Möller-Leimkühler et al., 2002).

Aunque los datos existentes sobre el consumo de alcohol muestran un mayor consumo en hombres que en mujeres, esta diferencia parece ir disminuyendo progresivamente en muchos países. Estos cambios en el patrón de consumo han sido relacionados con cambios en los roles sociales que implican una mayor igualdad de oportunidades entre hombres y mujeres y una mayor incorporación de la mujer a la vida pública, habiendo sido asociados, por ejemplo, a la incorporación de la mujer al trabajo fuera de casa, a la adopción de valores y comportamientos típicamente masculinos, o a la mayor libertad económica y de consumo de las mujeres (Díaz Geada, Busto Miramontes y Caamaño Isorna, 2018; Holmila y Raitasalo, 2005). Otros estudios indican que parece existir un cambio generacional respecto a la evolución de la identidad de género femenina y su relación con la percepción social del consumo de alcohol en mujeres. Tradicionalmente, el consumo de alcohol ha sido asociado a la masculinidad, a la construcción de lazos masculinos, a la agresión y a la conducta transgresora (Graham y Wells, 2003), siendo percibido socialmente como menos reprochable que el consumo femenino, considerado más como una amenaza a la feminidad tradicional y a la gestión de las tareas domésticas y de cuidado de la familia (de 
Visser y McDonnell, 2012). Algunos autores defienden que esta evolución, más que un efecto exclusivo de imitación por parte de las mujeres de los valores asociados a la masculinidad, podría explicarse por el desarrollo de nuevos modelos de feminidad donde el consumo de alcohol no es percibido de forma tan negativa como en el modelo tradicional. Así, por ejemplo, se ha señalado que las generaciones más jóvenes de mujeres perciben el consumo de alcohol de forma menos negativa para la identidad femenina que las mujeres de mayor edad, educadas en un modelo de identidad de género más tradicional (Simonen, Törrönen y Tigerstedt, 2013). Sin embargo, esta percepción parece estar ligada al consumo en determinados lugares o situaciones, como los espacios sociales o de ocio (Törrönen, Rolando y Beccaria, 2017). Desde este enfoque, investigaciones recientes han señalado la importancia de estudiar el papel combinado de la edad y de los roles de género en el inicio y mantenimiento del consumo de alcohol (Fernández Rodríguez, Dema Moreno y Fontanil Gómez, 2019).

A la luz de los datos revisados, la investigación parece confirmar que existen relaciones significativas entre el consumo de alcohol y la conformidad con ciertas normas de género, aspectos que tienen que ver con procesos de aprendizaje y socialización diferencial respecto a lo considerado masculino y femenino dentro de cada sociedad, apuntando a su relevancia en el ámbito del desarrollo de estrategias y programas de prevención e intervención. Los perfiles de morbilidad o mortalidad de cualquier país se relacionan con patrones de comportamientos de salud y la posibilidad de ser modificados promoviendo conductas saludables pasa por identificar estas creencias sociales en torno a los roles tradicionales masculinos y femeninos. Sin embargo, uno de los mayores retos a los que se enfrenta la investigación sobre esta temática es la dificultad de obtener resultados generalizables sobre el estudio de las normas de género debido a la influencia que sobre ellas tiene la cultura y la sociedad concreta en la que se evalúe. El desarrollo y la estandarización de las medidas de género y la adaptación a los distintos contextos culturales y geográficos, como es el caso de algunos de los cuestionarios utilizados en los trabajos revisados (CMNI o CFNI), es una tarea necesaria para continuar profundizando en el estudio de estas relaciones y poder generalizar y extraer conclusiones válidas de la investigación sobre la conformidad con las normas de género y su relación con variables relativas a la salud como el consumo de alcohol.

Entre las limitaciones de esta revisión se debe señalar que los resultados encontrados están limitados a los criterios de selección empleados y a las bases de datos utilizadas. Así, las publicaciones indexadas en otras bases de datos electrónicas, estudios escritos en otras lenguas, además del inglés y el español, o la revisión de otros tipos de investigaciones no publicadas en revistas especializadas, podrían haber sido omitidas del presente análisis. Sin embargo, y dada la escasez de investigación sobre el tema de estudio, la revisión realizada permite obtener una serie de conclusiones consistentes entre los estudios analizados, así como sobre las limitaciones y retos futuros de investigación.

Finalmente, y a modo de conclusión, podemos confirmar que ciertos aspectos negativos relacionados tradicionalmente con la masculinidad parecen actuar como factores de riesgo para el consumo de alcohol. Por el contrario, ciertas características asociadas a la feminidad y a una masculinidad positiva o asertiva parecen tener un efecto protector ante el consumo. La posibilidad de modificar ciertos patrones de creencias asociadas a la identidad y a las normas de género puede ser un aspecto relevante para el cambio y la modificación de ciertos comportamientos de riesgo para la salud. Asimismo, esta posibilidad plantea una serie de retos futuros de investigación que pasan por profundizar en mayor medida en el estudio de estas relaciones, desarrollar instrumentos de medida de género adecuados, y explorar nuevas hipótesis en relación al desarrollo de nuevos modelos de feminidad y masculinidad.

\section{Conflictos de intereses}

Los autores declaran no tener ningún conflicto de intereses.

\section{Referencias}

Alvanzo, A. A., Storr, C. L., La Flair, L., Green, K. M., Wagner, F. A. y Crum, R. M. (2011). Race/ethnicity and sex differences in progression from drinking initiation to the development of alcohol dependence. Drug and Alcohol Dependence, 118, 375-382. doi:10.1016/j.drugalcdep.2011.04.024.

Antill, J. K., Cunningham, J. D., Russell, G. y Thompson, N. L. (1981). An Australian Sex-Role Scale. Australian Journal of Psychology, 33, 169-183.

Ávila, J. y González, D. (2007). Diferencias de género en la enfermedad alcohólica. Adicciones, 19, 383-392. doi:10.20882/adicciones.297.

Bem, S. L. (1974). The measurement of psychological androgyny. Journal of Consulting and Clinical Psychology, 42, 155-162. doi:10.1037/h0036215.

Bem, S. L. (1981). Bem Sex Role Inventory professional manual. Palo Alto, CA: Consulting Psychologists Press.

Bergman, H., Bergman, I., Engelbrektsson, K., Holm, L., Johannesson, K. y Lindberg, S. (1988). Psykologhandbok del 1 (Manual for Psychologists: Part 1). Stockholm: The Magnus Huss Clinic, Karolinska Hospital.

Brabete, A. C. y Sánchez-López, M. P. (2012). How does the gender influence people's health? Data of a sample of Romanian people living in Spain. Procedia-Social and Behavioral Sciences, 33, 148-152. doi:10.1016/j.sbspro.2012.01.101. 
Brabete, A. C., Sánchez-López, M. P., Cuéllar-Flores, I. y Rivas-Díez, R. (2013). The impact of gender norms on alcohol and tobacco use at Romanians. Procedia-Social and Behavioral Sciences, 78, 230-234. doi:10.1016/j.sbspro.2013.04.285.

Bríñez-Horta, J. A. (2001). Diferencias de género en problemas con el alcohol, según el nivel de consumo. Adicciones, 13, 439-455. doi:10.20882/adicciones.559.

Collins, R. L., Parks, G. A. y Marlatt, G. A. (1985). Social determinants of alcohol consumption: The effects of social interaction and model status on the self-administration of alcohol. Journal of Consulting and Clinical Psychology, 53, 189-200. doi:10.1037/0022-006x.53.2.189.

Courtenay, W. H. (2000). Behavioral factors associated with disease, injury, and death among men: Evidence and implications for prevention. The Journal of Men's Studies, 9, 81-142. doi:10.3149/jmh.0103.281.

Cuéllar-Flores, I., Sánchez-López, M.P. y Dresch, V. (2011). El Inventario de Conformidad con las Normas de Género Masculinas (CMNI) en la población española. Anales de Psicología, 27, 170-178.

de Visser, R. y McDonnell, E. J. (2012). "That's OK. He's a guy": A mixed-methods study of gender double-standards for alcohol use. Psychology $\mathcal{E}$ Health, 27, 618-639. doi:10.1080/08870446.2011.61744.

Díaz Geada, A., Busto Miramontes, A. y Caamaño Isorna, F. (2018). Alcohol, tobacco and cannabis consumption in adolescents from a multicultural population (Burela, Lugo). Adicciones, 30, 264-270. doi:10.20882/adicciones.915.

Díaz-Mesa, E. M., García-Portilla, P., Fernández-Artamendi, S., Sáiz, P. A., Bobes, T., Casares, M. J., ... Bobes, J. (2016). Diferencias de género en la gravedad de la adicción. Adicciones, 28, 221-230. doi:10.20882/adicciones.829.

Ehlers, C. L., Gizer, I. R., Vieten, C., Gilder, A., Gilder, D. A., Stouffer, G. M., ... Wilhelmsen, K. C. (2010). Age at Regular Drinking, Clinical Course, and Heritability of Alcohol Dependence in the San Francisco Family Study: A Gender Analysis. The American Journal on Addictions, 19, 101-110. doi:10.1111/j.1521-0391.2009.00021.x.

Ely, M., Hardy, R., Longford, N. y Wadsworth, M. (1999). Gender differences in the relationship between alcohol consumption and drink problems are largely accounted for body water. Alcohol and Alcoholism, 34, 894-902. doi:10.1093/alcalc/34.6.894.

Fernández Rodríguez, M.A., Dema Moreno, S. y Fontanil Gómez, Y. (2019). The influence of gender roles in alcohol consumption: a qualitative study of adolescents and young adults in Asturias. Adicciones, 31, 260-273. doi:10.20882/adicciones.1003.

Good, G. E., Schopp, L. H., Thomson, D., Hathaway, S. L., Mazurek, M. O. y Sanford-Martens, T. C. (2008). Men with serious injuries: Relations among masculinity, age, and alcohol use. Rehabilitation Psychology, 53, 39-45. doi:10.1037/0090-5550.53.1.39.
Gordon, D. M., Hawes, S. W., Reid, A. E., Callands, T. A., Magriples, U., Divney, A., ... Kershaw, T. (2013). The many faces of manhood: Examining masculine norms and health behaviors of young fathers across race. American Journal of Men's Health, 7, 394-401. doi:10.1177/1557988313476540.

Graham, K. y Wells, S. (2003). Somebody's Gonna Get Their Head Kicked in Tonight Aggression among Young Males in Bars: A Question of Values? British Journal of Criminology, 43, 546-566. doi:10.1093/bjc/43.3.546.

Hensing, G. y Spak, F. (2009). Lack of leadership confidence relates to problem drinking in women: gender identity, heavy episodic drinking and alcohol use disorders in Swedish women. Alcohol and Alcoholism, 44, 626-633. doi:10.1093/alcalc/agp072.

Hensing, G., Spak, F., Thundal, K. L. y Östlund, A. (2003). Decreased risk of alcohol dependence and/or misuse in women with high self-assertiveness and leadership abilities. Alcohol and Alcoholism, 38, 232-238. doi:10.1093/ al-calc/agg058.

Holmila, M. y Raitasalo, K. (2005). Gender differences in drinking: why do they still exist? Addiction, 100, 17631769. doi:10.1111/j.1360-0443.2005.01249.x.

Hsu, K. y Iwamoto, D. K. (2014). Testing for measurement invariance in the Conformity to Masculine Norms-46 across White and Asian American college men: Development and validity of the CMNI-29. Psychology of Men $\mathcal{E}$ Masculinity, 15, 397-406. doi:10.1037/a0034548.

Iwamoto, D. K., Corbin, W., Lejuez, C. y MacPherson, L. (2014). College men and alcohol use: Positive alcohol expectancies as a mediator between distinct masculine norms and alcohol use. Psychology of Men $\mathcal{E}$ Masculinity, 15, 29-39. doi:10.1037/a0031594.

Iwamoto, D. K., Cheng, A., Lee, C. S., Takamatsu, S. y Gordon, D. (2011). "Maning" up and getting drunk: The role of masculine norms, alcohol intoxication and alcohol-related problems among college men. Addictive Behaviors, 36, 906-911. doi:10.1016/j.addbeh.2011.04.005.

Iwamoto, D. K. y Smiler, A. P. (2013). Alcohol makes you macho and helps you make friends: The role of masculine norms and peer pressure in adolescent boys' and girls' alcohol use. Substance Use Eं Misuse, 48, 371-378. doi:10.3109/10826084.2013.765479.

Kahler, C. W., Strong, D. R. y Read, J. P. (2005). Toward Efficient and Comprehensive Measurement of the Alcohol Problems Continuum in College Students: The Brief Young Adult Alcohol Consequences Questionnaire. Alcoholism: Clinical E Experimental Research, 29, 1180-1189. doi:10.1097/01.alc.0000171940.95813.a5.

Kaya, A., Iwamoto, D. K., Grivel, M., Clinton, L. y Brady, J. (2016). The role of feminine and masculine norms in college women's alcohol use. Psychology of Men E् Masculinity, 17, 206-214. doi:10.1037/men0000017. 
Kulis, S., Marsiglia, F. F., Lingard, E. C., Nieri, T. y Nagoshi, J. (2008). Gender identity and substance use among students in two high schools in Monterrey, Mexico. Drug and Alcohol Dependence, 95, 258-268. doi:10.1016/j. dru-galcdep.2008.01.019.

Kulis, S., Marsiglia, F. F. y Nagoshi, J. L. (2010). Gender roles, externalizing behaviors, and substance use among Mexican-American adolescents. Journal of Social Work Practice in the Addictions, 10, 283-307. doi:10.1080/1533 256X.2010.497033.

Levant, R. F., Smalley, K. B., Aupont, M., House, A. T., Richmond, K. y Noronha, D. (2007). Initial validation of the Male Role Norms Inventory-Revised (MRNI-R). The Journal of Men's Studies, 15, 83-100. doi:10.3149/jms.1501.83.

Liberati, A., Altman, D. G., Tetzlaff, J., Mulrow, C., Gotzsche, P. C., Ioannidis, J. P., ... Moher, D. (2009). The PRISMA statement for reporting systematic reviews and meta-analyses of studies that evaluate health care interventions: explanation and elaboration. PLoS Medicine, 6, e1000100. doi:10.1371/journal.pmed.1000100.

Liu, W. M. y Iwamoto, D. K. (2007). Conformity to masculine norms, Asian values, coping strategies, peer group influences and substance use among Asian American men. Psychology of Men $\mathcal{E}$ Masculinity, 8, 25-39. doi:10.1037/1524-9220.8.1.25.

Lye, D. N. y Waldron, I. (1998). Relationships of substance use to attitudes toward gender roles, family and cohabitation. Journal of Substance Abuse, 10, 185-198. doi:10.1016/s0899-3289(99)80133-3.

Mahalik, J. R., Locke, B. D., Ludlow, L. H., Diemer, M. A., Scott, R. P., Gottfried, M. y Freitas, G. (2003). Development of the Conformity to Masculine Norms Inventory. Psychology of Men E Masculinity, 4, 3-25. doi:10.1037/1524-9220.4.1.3.

Mahalik, J. R., Morray, E. B., Coonerty-Femiano, A., Ludlow, L. H., Slattery, S. M. y Smiler, A. (2005). Development of the Conformity to Feminine Norms Inventory. Sex Roles, 52, 417-435. doi:10.1007/s11199-005-3709-7.

Mahalik, J. R., Burns, S. M. y Syzdek, M. (2007a). Masculinity and perceived normative health behaviors as predictors of men's health behaviors. Social Science $\mathcal{E}$ Medicine, 64, 2201-2209. doi:10.1016/j.socscimed.2007.02.035.

Mahalik, J. R., Levi-Minzi, M. y Walker, G. (2007b). Masculinity and health behaviors in Australian men. Psychology of Men E् Masculinity, 8, 240-249. doi:10.1037/15249220.8.4.240.

Míguez, M. C. y Permuy, B. (2017). Características del alcoholismo en mujeres. Revista de la Facultad de Medicina, 65, 15-22. doi:10.15446/revfacmed.v65n1.57482.

Mokdad, A. H., Marks, J. S., Stroup, D. F. y Gerberding, J. L. (2004). Actual causes of death in the United States, 2000. JAMA, 291, 1238-1245. doi:10.1001/jama.291.10.1238.

Möller-Leimkühler, A. M., Schwarz, R., Burtscheidt, W. y Gaebel, W. (2002). Alcohol dependence and gender-ro- le orientation. European Psychiatry, 17, 1-8. doi:10.1016/ S0924-9338(02)00624-7.

Nathanson, C. A. (1975). Illness and the feminine role: a theoretical review. Social Science Eै Medicine, 9, 57-62. doi:10.1016/0037-7856(75)90094-3.

Observatorio Español de las Drogas y las Adicciones (2017). Alcohol, tabaco y drogas ilegales en España: Informe 2017. Encuesta sobre alcohol y drogas en España (EDADES) 1995-2015. Madrid: Ministerio de Sanidad, Servicios Sociales e Igualdad. Recuperado de htttp:/ /www.pnsd.mscbs.gob.es/profesionales/ sistemasInformacion/sistemaInformacion/pdf/2017_Informe_EDADES.pdf.

O’Neil, J. M., Helms, B. J., Gable, R. K., David, L. y Wrightsman, L. S. (1986). Gender-Role Conflict Scale: College men's fear of femininity. Sex Roles, 14, 335-350. doi:10.1007/BF00287583.

Organización Mundial de la Salud (2016). Life expectancy and healthy life expectancy. Recuperado de http:/ / apps.who.int/gho/data/view.main.SDG2016LEXREGv?lang=en.

Parent, M. C. y Moradi, B. (2009). Confirmatory factor analysis of the Conformity to Masculine Norms Inventory and development of the Conformity to Masculine Norms Inventory-46. Psychology of Men $\mathcal{E}$ Masculinity, 10, 175-189. doi:10.1037/a0015481.

Parent, M. C. y Moradi, B. (2010). Confirmatory factor analysis of the conformity to feminine norms inventory and development of an abbreviated version: the CFNI-45. Psychology of Women Quarterly, 34, 97-109. doi:10.1111/j.1471-6402.2009.01545.x.

Polderman, T. J. C., Benyamin, B., de Leeuw, C. A., Sullivan, P. F., van Bochoven, A., Visscher, P. M. y Posthuma, D. (2015). Meta-analysis of the heritability of human traits based on fifty years of twin studies. Nature Genetics, 47, 702-709. doi:10.1038/ng.3285.

Ricciardelli, L. A., Williams, R. J. y Kiernan, M. J. (1998). Relation of drinking and eating to masculinity and femininity. The Journal of Social Psychology, 138, 744-752. doi:10.1080/00224549809603259.

Runge, T. E., Frey, D., Gollwitzer, P. M., Helmreich, R. L. y Spence, J. T. (1981). Masculine (instrumental) and feminine (expressive) traits. A comparison between students in the United States and West Germany. Journal of Cross-Cultural Psychology, 12, 142-162. doi:10.1177/0022022181122002.

Sánchez-Autet, M., Garriga, M., Zamora, F. J., González, I., Usall, J., Tolosa. L., ... Arranz, B. (2018). Screening of alcohol use disorders in psychiatric outpatients: influence of gender, age, and psychiatric diagnosis. Adicciones, 30, 251-263. doi:10.20882/adicciones.885.

Sánchez-López, M. P. (2013). La salud desde la perspectiva de género: el estado de la cuestión. En M. P. Sánchez-López (Eds.), La salud de las mujeres (pp.17-40). Madrid, España: Síntesis. 
Sánchez-López, M. P., Cuéllar-Flores, I. y Dresch, V. (2012). The impact of gender roles on health. Women $\mathcal{E}$ Health, 52, 182-196. doi:10.1080/03630242.2011.652352.

Sánchez-López, M. P. y Limiñana-Gras, R. M. (2017). Health from a gender perspective. En M. P. Sánchez-López y R. M. Limiñana-Gras (Eds.), The Psychology of Gender and Health (pp.1-52). London: Elsevier. doi:10.1016/b978-012-803864-2.00001-8.

Sánchez-López, M. P., Rivas-Díez, R. y Cuéllar-Flores, I. (2013). Masculinity and Femininity as predictors of tobacco and alcohol consumption in Spanish university students. Health and Addictions, 13, 15-22. doi:10.21134/ haaj.v13i1.189.

Sánchez-López, M. P., Saavedra, A. I., Dresch, V. y Limiñana-Gras, R. M. (2014). Conformity to Traditional Gender Norms in a Feminized Occupation: The Influence on Health Behaviors. Health, 6, 2775-2789. doi:10.4236/ health.2014.620317.

Simonen, J., Törrönen, J. y Tigerstedt, C. (2013) . Femininities of drinking among Finnish and Swedish women of different ages. Addiction: Research Ev Theory, 22, 98-108. doi:10.3109/16066359.2013.779676.

Spence, J. T. y Helmreich, R. L. (1978). Masculinity and femininity: their psychological dimensions, correlates, and antecedents. Austin, TX: University of Texas Press.

Thompson, E. H. y Pleck, J. H. (1986). The structure of Male Role Norms. American Behavioral Scientist, 29, 531543. doi:10.1177/000276486029005003.

Törrönen, J., Rolando, S. y Beccaria, F. (2017). Masculinities and femininities of drinking in Finland, Italy and Sweden: Doing, modifying and unlinking gender in relation to different drinking places. Geoforum, 82, 131140. doi:10.1016/j.geoforum.2017.04.005.
Uy, P. J., Massoth, N. A. y Gottdiener, W. H. (2014). Rethinking male drinking: Traditional masculine ideologies, gender-role conflict, and drinking motives. Psychology of Men E् Masculinity, 15, 121-128. doi: 10.1037/a0032239.

Van Gundy, K., Schieman, S., Kelley, M. S. y Rebellon, C. J. (2005). Gender role orientations and alcohol use among Moscow and Toronto adults. Social Science E Medicine, 61, 2317-2330. doi:10.1016/j.socscimed.2005.07.033.

Vaughan, E. L., Wong, Y. J. y Middendorf, K. G. (2014). Gender roles and binge drinking among Latino emerging adults: a latent class regression analysis. Psychology of Addictive Behaviors, 28, 719-726. doi:10.1037/a0037406.

Verbrugge, L. M. (1982). Sex differentials in health. Public Health Reports, 97, 417-437.

Verbrugge, L. M. (1989). The twain meet: empirical explanations of sex differences in health and mortality. Journal of Health and Social Behavior, 30, 282-304. doi:10.2307/2136961.

Verhulst, B., Neale, M. C. y Kendler, K. S. (2014). The heritability of alcohol use disorders: a meta-analysis of twin and adoption studies. Psychological Medicine, 45, 10611072. doi:10.1017/s0033291714002165.

White, H. R. y Labouvie, E. W. (1989). Rutgers Alcohol Problem Index. PsycTESTS Dataset. doi:10.1037/t00517000 .

Williams, R. J. y Ricciardelli, L. A. (1999). Gender congruence in confirmatory and compensatory drinking. The Journal of Psychology, 133, 323-331. doi:10.1080/00223989909599745.

Williams, R. J. y Ricciardelli, L. A. (2001). Sex-role traits and the comorbidity of symptoms of disordered eating and problem drinking. Eating Behaviors, 2, 67-77. doi:10.1016/s1471-0153(00)00024-6. 